\title{
Perspective
}

PERSPECTIVE Actualité en histoire de l'art

$1 \mid 2020$

Japon

\section{Entretien avec Jean-Claude Lebensztejn}

par Atsushi Miura, introduction par Yosuke Morimoto

Atsushi Miura, Yosuke Morimoto et Jean-Claude Lebensztejn

\section{CpenEdition}

Journals

Édition électronique

URL : http://journals.openedition.org/perspective/17813

DOI : $10.4000 /$ perspective. 17813

ISSN : 2269-7721

Éditeur

Institut national d'histoire de l'art

\section{Édition imprimée}

Date de publication : 5 juin 2020

Pagination : 89-110

ISBN : 978-2-917902-89-9

ISSN : $1777-7852$

Référence électronique

Atsushi Miura, Yosuke Morimoto et Jean-Claude Lebensztejn, « Entretien avec Jean-Claude

Lebensztejn », Perspective [En ligne], 1 | 2020, mis en ligne le 30 décembre 2020, consulté le 25 janvier

2021. URL : http://journals.openedition.org/perspective/17813 ; DOI : https://doi.org/10.4000/

perspective. 17813 


\section{Entretien avec Jean-Claude Lebensztejn}

par Atsushi Miura, introduction par Yosuke Morimoto

\section{Jean-Claude Lebensztejn au Japon}

On aurait tort de croire pouvoir localiser Jean-Claude Lebensztejn en quelque lieu terrestre que ce soit, au Japon ou ailleurs, peu importe. Ce Doctor Angelicus ${ }^{1}$ déjoue, déplace les frontières en tout genre, tout en interrogeant avec une vigilance extrêmement aiguë les échos et les répercussions que les distances produisent, et qui prêtent, nécessairement, pour le pire ou le meilleur, au malentendu.

Textuellement parlant au moins, notre pays ne semble pas occuper chez lui une place particulièrement privilégiée, aucun artiste, aucune œuvre nipponne n'ayant fait l'objet d'une étude à part entière. En revanche la référence japonaise vient nous surprendre çà et là, où l'on s'y attend peu : cette citation d'un propos ludique et émouvant de Hokusai au début du mythique Zigzag² ; cette photo d'un melon à demi personnifié qui s'égaie sur une boîte en carton ondulé, prise peut-être chez un marchand de primeurs de HidaTakayama (au centre du Japon) pour décorer la couverture de Déplacements ${ }^{3}$; et surtout cette inoubliable performance de lecture que Lebensztejn a bien voulu improviser au cours d'une conférence tenue pendant le bel été de 2014, à Tōkyō, en déclamant pour nous la fameuse légende surplombant l'une des acmés érotiques de Hokusai, Le Rêve de la femme du pêcheur.

En épilogue à Miaulique (qui cite un jésuite, Luís Fróis, perplexe devant les contrastes radicaux entre les deux cultures du Japon et de l'Europe), on peut lire ceci :

J'avais demandé un séjour d'étude dans un institut français au Japon. Les instances de décision me firent savoir que mon projet ne correspondait pas aux orientations de cet institut. J'aurais dû le prévoir : un organisme créé pour favoriser les échanges culturels ne peut accepter un projet soulignant les mésententes ${ }^{4}$.

Le livre fut imprimé en octobre 2002 ; l'auteur prévoyait-il alors que l'entretien ici reproduit dans sa version originale française se déroulerait deux mois plus tard, le 21 décembre 2002 ?

Conduit par Atsushi Miura, transcrit et traduit en japonais par moi-même - dixhuit ans après donc, mais je peux me rappeler chacun des coups de téléphone que 
l'interviewé me donna alors pour pointer, avec son élégante précipitation, tous les malentendus que je provoquais -, cet entretien fut publié en 2003 dans le numéro spécial de la revue 『西洋美術史研究』[Études sur l'histoire de l'art occidental] consacré au thème du parergon. Non seulement il permit au lectorat japonais de constater toute la singularité de la personnalité de Lebensztejn demeurée jusqu'alors quelque peu énigmatique, autant que l'envergure et la diversité de ses travaux qui nous fascinaient depuis les années $1970^{5}$, mais il marqua le début d'une série d'échanges plus concrets : en plus de conférences données à Tōkyō et à Kyōto en 2009, puis à nouveau à Tōkyō en 2014, Jean-Claude Lebensztejn s'est mis à nous écrire, à nous autres chercheurs japonais, de façon amicale et constante, nous faisant ainsi partager ses nouveaux textes, ses découvertes et différents " faits divers ", allant même jusqu'à prodiguer de précieux conseils aux plus jeunes étudiants qui lui ont rendu visite à Paris. Et c'est sans compter plusieurs traductions qui se firent jour depuis (entre autres celles des Études cézanniennes en 2009 et de Miaulique en $2014^{6}$; fig. 1).

Ayant eu l'expérience de traduire ce dernier ouvrage, ai-je le droit de revenir à l'évidence : l'écriture de Lebensztejn constitue un beau défi pour le traducteur. Comme elle est dépouillée de tout néologisme et idiotisme, l'écueil n'est pas d'ordre terminologique ou syntagmatique. La tâche en revanche est de reproduire, dans la mesure du possible, à la fois la fluidité de son mouvement et son caractère syncopé, ses à-pic. L'auteur s'attache aux détails (" Je m'attache à des détails qui font problème ", dit-il dans l'entretien) et ne se plaît guère à les détailler. Ainsi aime-t-il citer des noms et des phrases, parfois d'un seul trait long et prolongé, mais bien souvent sans en disserter doctement, pour vite passer à d'autres, comme s'il préférait nous les livrer tous dans leur concordia discors ${ }^{7}$. Une telle permanente ritournelle textuelle nous conduit à songer à cet idéal nommé sprezzatura. Mais si, de celle-ci, l'auteur lui-même se demande " comment écrire sans lui manquer, la manquer, en manquer ${ }^{8}$ ", qui, à vouloir le traduire, prétendrait s'épargner un pareil manquement ?

Quelles leçons avons-nous pu recevoir de Lebensztejn ? À tant de qualités reconnues par tous ses lecteurs d'ici ou de partout ailleurs - sa précision philologique, son agilité dans l'analyse des images, son esprit en perpétuel déplacement, pour n'évoquer qu'eux -, je me borne à ajouter juste une petite chose : il nous a appris la valeur de l'impertinence, à condition que celle-ci soit accompagnée d'une "innervation " si méticuleuse qu'elle va jusqu'à dépasser la limite de l'étudié et du spontané, du réel et du rêvé qui en émane.

Je termine, provisoirement, en insistant sur ce simple fait : Jean-Claude Lebensztejn nous fascine, nous comme vous toutes et tous, par sa présence, d'autant plus irremplaçable qu'insaisissable. Une telle fascination, " il est vrai, suppose une part d'assimilation, et par conséquent de transformation, de distorsion et de rejet, mais cette part doit rester le secret de l'échange ${ }^{9} "$. Fors ce secret, souhaité-je, l'histoire de notre échange, mais aussi celle des échanges qu'il suscite autour de lui, restera toujours ouverte.

[Yosuke Morimoto]

- Atsushi Miura. La revue Études sur l'histoire de l'art occidental traite dans son numéro 9 du thème du parergon, il m'a donc semblé tout naturel, pour l'interview de ce numéro spécial, d'inviter monsieur Lebensztejn. Vous êtes sans aucun doute l'historien de l'art - si vous me permettez de vous appeler ainsi - le plus important, et le plus pertinent à l'égard de cette problématique. À travers vos nombreux ouvrages stimulants, et surtout dans les Annexes (Bruxelles, La Part de l'OEil, 1999) - dont le titre est déjà symbolique -, vous avez 
pratiqué, dans la discipline de l'histoire de l'art, la pensée théorique de Jacques Derrida, notamment sa Vérité en peinture (1978), pour mettre en évidence une nouvelle possibilité méthodologique dans notre domaine. En ce sens, on pourrait même dire que vous êtes un anti-historien de l'art et un méta-historien de l'art. Mais, avant d'entrer directement au cœur du problème, je voudrais faire un petit détour sur votre formation et, pour cela, évoquer un nom très important: Meyer Schapiro. Vous avez traduit en français trois de ses articles, et l'on trouve aussi dans le texte de Derrida l'indication du nom de Schapiro. Quels sont les apports de Schapiro dans votre formation?

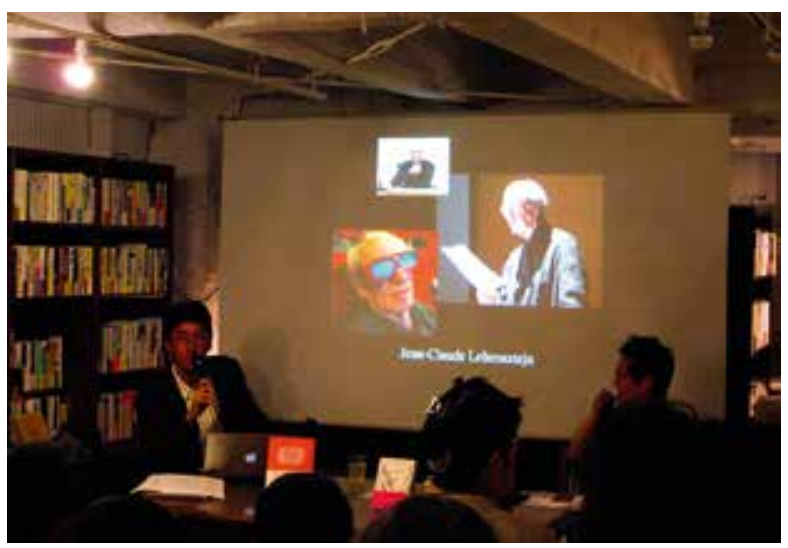

1. Yosuke Morimoto présente sa traduction de Miaulique, Tōkyō, en 2014 : 『猫の音楽』森元庸介訳、 勁草書房、2014年。.

- Jean-Claude Lebensztejn. J'ai rencontré Schapiro en 1968. Je voulais traduire pour un cercle français de théorie de l'art animé par Hubert Damisch l'article "Léonard et Freud ${ }^{10}$ ", et j'ai écrit à Schapiro, qui m’a répondu : cet article est déjà pris par Jean-Bertrand Pontalis, qui doit le publier avec une nouvelle traduction du texte de Freud "Un souvenir d'enfance de Léonard de Vinci ». Il ajoutait : si vous voulez, je vous donne un texte inédit sur la sémiotique des arts visuels. Il m’a fait le plaisir, sans me connaître, de me donner ce texte à traduire. Je l'ai traduit. Nous avons très longuement discuté de la traduction, parce qu'il était très minutieux, et que je le suis moi-même. J'ai aussi entendu des conférences de Schapiro, et je l'ai beaucoup lu. Je n'ai jamais suivi ses cours à Columbia, mais j'ai pas mal discuté avec lui en privé. J'ai trouvé extrêmement important son article sur la sémiotique des arts visuels. Il a été publié plus tard, en anglais en 1969, dans Semiotica, en français dans Critique en $1973^{11}$, puis dans le volume d'essais de Schapiro paru chez Gallimard en 1982 ; à cette occasion, j'ai pu traduire deux autres textes de Schapiro, le texte sur Léonard et Freud (qui n'avait pas été en fait publié par Pontalis) et le texte sur Seurat. C'étaient les trois textes qui m'intéressaient le plus parmi ceux que l'on m'avait proposé de traduire ${ }^{12}$.

- Atsushi Miura. Le problème de Schapiro nous amène directement au problème de Derrida, qui cite deux fois le nom de Schapiro, dans le chapitre sur le «Parergon » et dans le chapitre intitulé « Restitutions", toujours par rapport à votre nom.

- Jean-Claude Lebensztejn. Dans le cas de «Parergon », oui. Dans le cas de « Restitutions», non. Il s'agissait d'un rapport à Heidegger.

- Atsushi Miura. Mais dans la dédicace, on lit : « à J. C. ... sztejn » ?

- Jean-Claude Lebensztejn. Oui, c'est exact. Je pense que c'est une allusion au fait que Schapiro avait dédié son texte sur Heidegger à Kurt Goldstein ${ }^{13}$.

- Atsushi Miura. J'ai l'impression qu'il y avait une sorte de triangle Schapiro-LebensztejnDerrida à cette époque. 


\section{DERRIDA LA VÉRITÉ ENPEINTURE}

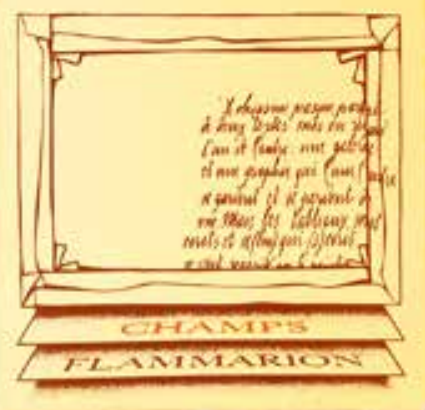

2. Jacques Derrida, La vérité en peinture, Paris, Flammarion, 1978
- Jean-Claude Lebensztejn. Mais ce n'est pas un triangle, dans la mesure où je n'étais pas un angle... Disons que les auteurs qui ont le plus occupé mon horizon intellectuel au moment de mes études, étaient, dans le domaine de l'histoire de l'art, Schapiro, et dans le domaine théorique, Derrida.

- Atsushi Miura. Vous avez alors participé au séminaire de Derrida ?

- Jean-Claude Lebensztejn. J'ai assisté à des séminaires de Derrida, particulièrement, en 1973-1974, au séminaire sur Kant et sa troisième Critique. Mais l'essentiel de ma réflexion sur les questions du parergon était déjà en place. Le texte sur le musée, le texte sur le fauvisme - un texte méthodologique sur la terminologie de l'histoire de l'art - et le texte sur la signature étaient écrits ou pensés au moment où Derrida donnait son séminaire sur Kant. Mais il faut dire que dans le cas de Derrida, le concept de parergon était en rapport avec d'autres concepts qu'il avait utilisés antérieurement, et particulièrement avec le concept de «supplément » dans la Grammatologie.

- Atsushi Miura. Et le mot parergon est historiquement fondé depuis Pline l'Ancien...

- Jean-Claude Lebensztejn. Tout à fait, c'est de là que Kant l'utilise dans sa troisième Critique, où Derrida l'a puisé, si I'on peut dire. Kant y employait le mot parerga, au pluriel.

- Atsushi Miura. En relisant la troisième Critique de Kant, Derrida élargit la connotation du mot parergon. Je cite :

[N]i œuvre (ergon), ni hors-d'œuvre, ni dedans ni dehors, ni dessus ni dessous, il déconcerte toute opposition, mais ne reste pas indéterminé et donne lieu à l'œuvre. Il n'est plus seulement autour d'elle. Ce qu'il met en place - les instances du cadre, du titre, de la signature, de la légende, etc. - ne laisse plus de déranger l'ordre interne du discours sur la peinture, ses œuvres, son commerce, ses évaluations, ses plus-values, sa spéculation, son droit et ses hiérarchies ${ }^{14}$.

N'y a-t-il pas déjà là un parergon concret et un parergon abstrait ?

- Jean-Claude Lebensztejn. Le parergon déjoue tous les dualismes, que Derrida déconstruit à cette occasion, y compris l'opposition du concret et de l'abstrait (fig. 2). Je trouve que le positivisme de l'historien de l'art qui s'occupe du cadre comme donnée matérielle reste dans ce schéma dualiste, et l'idée même de parergon doit déconstruire le cadre. Celui-ci est à la fois concret et abstrait, au-delà de l'opposition entre le concret et l'abstrait, et même au-delà de l'idée d'au-delà.

\section{- Atsushi Miura. Avez-vous beaucoup dialogué à l'époque avec Derrida ?}

- Jean-Claude Lebensztejn. Réellement, oui. Mais, comme je l'ai dit, ce n'est pas à la suite de ce séminaire que j'ai commencé à m'interroger sur ces questions, en particulier sur la question du cadre. Je me les suis posées aussi à d'autres occasions, comme dans l'étude sur Matisse, parue dans Critique en juillet-août 1974, mais écrite plus tôt. Et d'autres textes, 
sur Frank Stella, qui ont été publiés au début des années 1970, tournaient également autour de la question du cadre et de la limite.

- Atsushi Miura. Et nous arrivons à vos Annexes. Je cite encore une fois :

Ces textes, dont la rédaction s'échelonne entre 1967 et 1986, n'appellent pas de commentaire. Comme le titre l'indique, ils tournent autour des à-côtés de l'œuvre d'art, ses hors-d'œuvre - ce que Derrida relisant Kant a appelé ses parerga. Certains sont abstraits (les appellations, catégories, écoles), d'autres concrets (la signature, le cadre, l'accrochage), mais partout la logique affective du parergon opère un glissement de l'abstrait au concret et retour, de la périphérie au centre et retour : il n'est pas possible d'isoler une essence de l'art séparée de ses abords superficiels, tels ici, par exemple, le maquillage, la nonchalance ${ }^{15}$.

Vous avez donc classé vos articles en trois catégories : le concret et l'abstrait, et le maquillage et la nonchalance sont un peu à côté.

- Jean-Claude Lebensztejn. Disons que ces termes restent marqués par ce que Derrida appelle "paléonymie » - un vieux nom utilisé pour désigner une nouvelle chose. Je pense que cette idée du concret et de l'abstrait, de l'entre-deux, est déjà travaillée par l'idée de parergon. Apparemment, des catégories comme le fauvisme et d'autres appellations d'écoles sont d'ordre abstrait ; et, apparemment, des choses comme le cadre, la signature ou l'accrochage sont des objets concrets. Mais cette opposition n'est pas rigoureuse. Les appellations sont travaillées par le corps d'un texte qui est lui aussi concret. Et le cadre, la signature et l'accrochage sont travaillés par des données théoriques, sans lesquelles ils n'ont pas lieu d'être. On ne peut séparer le cadre concret de toutes les idéologies qui s'y sont déposées.

- Atsushi Miura. Commençons par ce que nous pourrions appeler provisoirement le parergon « concret». Vous avez écrit sur la signature et sur le cadre : deux notions qui renvoient, j'imagine, à l'espace pictural de la

3. Nicolas Poussin, Les Israélites recueillant la manne dans le désert, 1637-1639, Paris, musée du Louvre, inv. 7275.

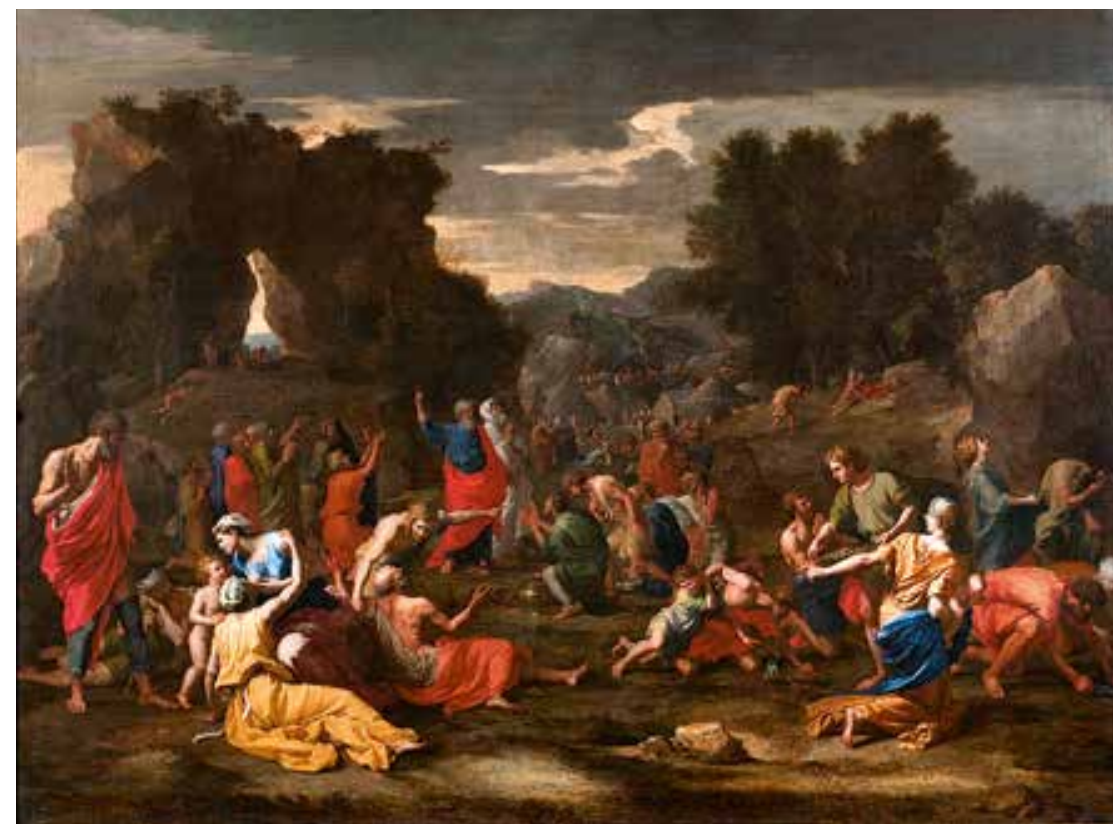




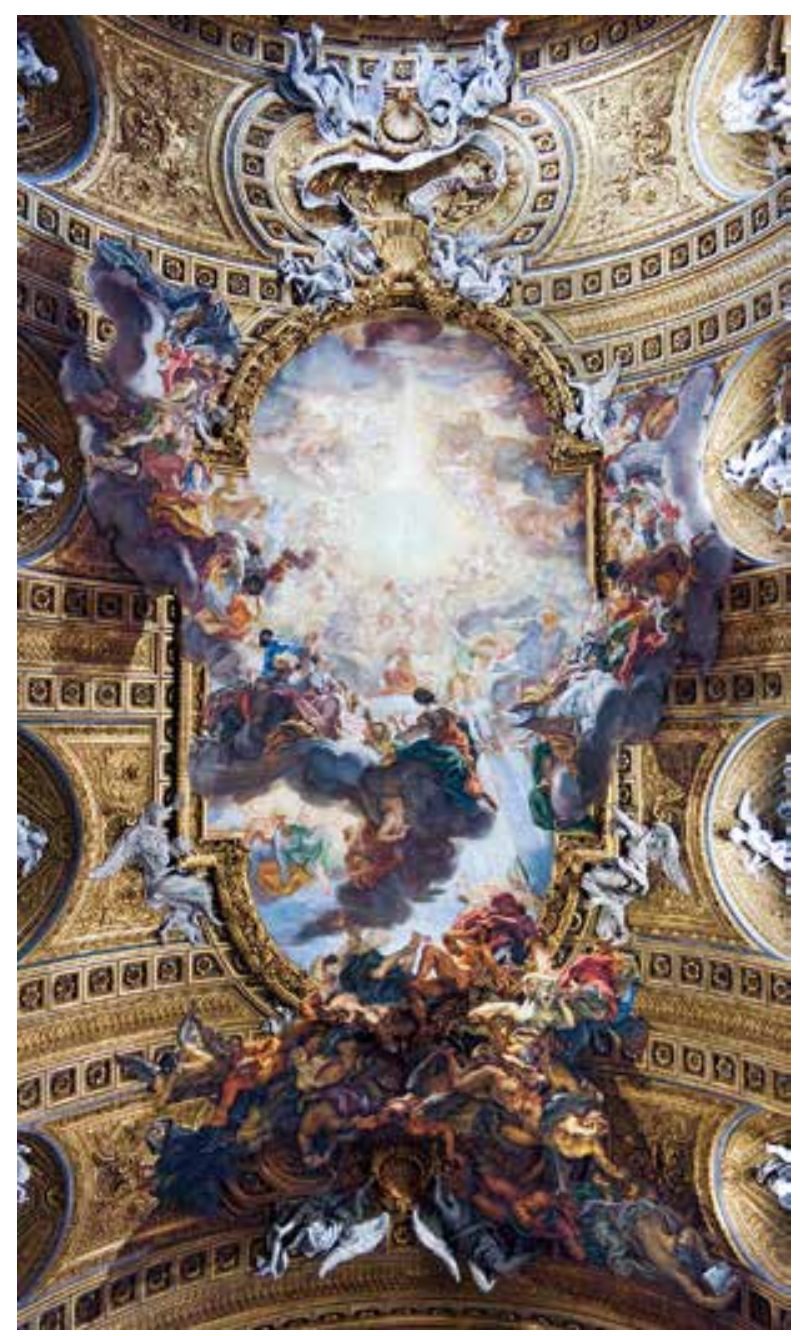

4. Giovanni Battista Gaulli, Le Triomphe du Nom de Jésus, 1672-1683, Rome, chiesa del Santissimo Nome di Gesù. représentation classique, et qui représentent, en tant que tels, une spécificité de l'art occidental et de la séparation qu'il suppose entre un dedans et un dehors.

- Jean-Claude Lebensztejn. Oui ; mais l'espace de la représentation classique est travaillé par une force adverse, que l'on voit à l'œuvre dans le trompe-l'œil, dans le plafond baroque, dans I'idée romantique d'une œuvre d'art totale (Gesamtkunstwerk), toutes sortes de choses qui travaillent cette opposition du dedans et du dehors.

Assurément il y a une position classique du cadre comme séparation entre le dedans et le dehors. Dans une célèbre lettre sur La Manne (fig. 3) à Chantelou, Poussin lui dit de donner à son tableau un cadre qui sépare bien, mais qui en même temps soit discret - une corniche « dorée d'or mat tout simplement ${ }^{16}$ ». Cette double exigence est importante. Il faut qu'un cadre n'attire pas l'attention sur lui ; en même temps, il faut qu'il soit efficace, c'est-à-dire qu'il sépare ce que Poussin appelle les « espèces ». (" Espèces » est un terme de la philosophie traditionnelle qui désigne ce qu'on appelle eidôla chez les matérialistes grecs, ou simulacra à l'époque de Lucrèce ${ }^{17}$. Le terme a été employé par exemple dans la conception aristotélicienne de la vision ; il désigne, entre l'objet et l'œil, les images qui viennent de l'objet pour frapper l'œil, ou bien qui viennent de l'œil pour frapper l'objet.) Poussin veut donc distinguer ce qu'il appelle les espèces de la peinture des espèces environnantes. Le cadre a cette fonction de séparation, mais de séparation discrète. À

l'époque de Poussin, on pouvait trouver des cadres très lourds, mais qui, en même temps, ne séparaient pas : par exemple dans les plafonds baroques, comme celui de l'église du Gesù à Rome (fig. 4), un peu postérieur à Poussin, où les figures débordent du cadre. Là, il y a transgression de la peinture dans l'espace du dehors. II s'agit de montrer l'universalité du nom de Jésus. Cela fait partie de la rhétorique baroque.

- Atsushi Miura. Vous avez aussi écrit sur la signature des peintres, c'est-à-dire les lettres dans l'image.

- Jean-Claude Lebensztejn. Oui, c'était pour un séminaire d'André Chastel, contemporain du séminaire de Derrida sur le parergon. Dans la signature peinte, on trouve toutes sortes de manières de faire travailler le texte et l'image, par exemple en inscrivant la signature 
en forme de trompe-l'œil, dans un des objets de l'image. On ne sait pas si la signature signe I'espace représenté ou l'espace représentant. II y a là toutes sortes de glissements entre le dedans et le dehors.

- Atsushi Miura. Parmi les parerga « concrets », vous n'avez pas écrit sur le problème du titre.

- Jean-Claude Lebensztejn. Non, je n'ai pas écrit là-dessus. Mais j'ai écrit un texte sur Brice

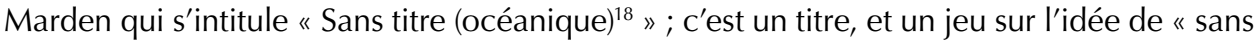
titre », qui peut être un titre, et pas un titre. Et « océanique » désigne l'espace entre deux continents, un espace maritime, mais aussi une terre sous la mer. Et cette terre invisible, sous-marine, relie deux continents séparés par l'océan. Ces deux espaces sont séparés, mais ils ne sont pas entièrement séparés.

\section{- Atsushi Miura. Et si vous aviez une occasion d'écrire sur le titre ?}

- Jean-Claude Lebensztejn. La plupart du temps, la peinture n'en porte pas, mais dans le cas de la gravure classique, l'image, généralement - pas toujours - porte un titre, au-dessous de l'image le plus souvent. Dans le cas de la peinture, normalement le tableau lui-même ne porte pas de titre ; celui-ci est inscrit sur le cadre, ou sur le mur, ou dans le livret. C'est la règle classique, mais elle peut être défaite dans les deux sens (fig. 5).

- Atsushi Miura. Je passe à la question des parerga " abstraits " : vous avez écrit un article intitulé "Sol ", très intéressant pour moi parce qu'il remet en cause, non seulement ces appellations consacrées de "fauvisme » et d'« expressionnisme ", mais aussi, très audacieusement, I'histoire de l'art elle-même et surtout sa méthode enracinée dans l'idéologie bourgeoise. Cet article était-il impubliable à cette époque-là ?

- Jean-Claude Lebensztejn. Oui, c'était impubliable. Cet article m'avait été demandé par Pierre Francastel au moment où je suivais son séminaire à l'École pratique des hautes études. Il m’a proposé en 1966 de faire quelque chose pour les Annales. L'occasion était une exposition qui venait d'avoir lieu à Paris sur le fauvisme français et les débuts de

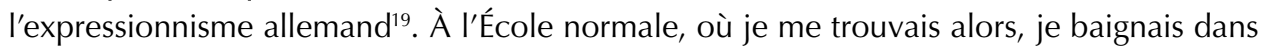
une pensée théorique de gauche, et quand j'ai fini ce texte, qui était très long, je l'ai porté à Paris - j'étais aux États-Unis à cette époque-là, au moment des événements de 1968 à Francastel, qui a déclaré que c'était impubliable. Il me l'a donc rendu. Et je l'ai publié quelques années plus tard dans une revue de réflexion de l'École normale, qui n'a eu que deux ou trois numéros - je ne sais plus -, Scolies (un titre donné par Derrida), à un moment où l'on essayait de ramasser les retombées de la pensée révolutionnaire, et qui a publié cela en deux parties dans deux numéros, parce que c'était trop long. Je me rappelle qu'à la fin de la première partie, qui était entièrement négative, j'ai vu Chastel, qui m'a dit : je vous attends au tournant.

- Atsushi Miura. Cette histoire est très intéressante. La situation concernant la méthode de l'histoire de l'art a-t-elle changé depuis lors?

- Jean-Claude Lebensztejn. Pas vraiment. Le mieux, c'est qu'on a commencé à faire connaître la grande tradition de l'histoire de l'art germanique, qui était inconnue en France à ce momentlà. Depuis, on s'est mis à publier les grands noms, Erwin Panofsky et Heinrich Wölfflin qui 
était déjà un peu connu, puis Aloïs Riegl et Aby Warburg qui est, maintenant, celui qui fait le plus travailler de jeunes historiens de l'art. Ce qui est positif, c'est cette ouverture à ce qui est, à mon avis, la seule école d'histoire de l'art véritablement importante au XXe siècle.

\section{- Atsushi Miura. Mais vous avez choisi Meyer Schapiro.}

- Jean-Claude Lebensztejn. Schapiro était à ce moment-là le seul à travailler de manière concrète et en même temps théorique sur les objets qui m'intéressaient. Je connaissais un peu les textes de Panofsky et ceux de Wölfflin, déjà traduits en français, mais pas ceux de Riegl et de Warburg, traduits très tardivement, dans les années 1990 (un essai de Riegl avait été traduit par Daniel Arasse dans le second volume de Scolies, en 1972). Mais Schapiro, parce qu'il était vivant et en pleine activité dans les années 1960, était la seule figure qui pouvait à l'époque représenter pour moi quelque chose de fondamental. Il était capable de voir l'objet artistique d'une manière qui ne soit pas confuse - qui ne soit pas mystifiée et mystifiante.

- Atsushi Miura. Dans ses textes, on trouve toujours à la fois une rigueur logique et une sensibilité aiguë aux détails.

- Jean-Claude Lebensztejn. Oui. En même temps, ce texte sur la sémiotique ${ }^{20}$ faisait sortir I'histoire de l'art théoricienne du carcan linguistique. Il faut dire qu'à l'époque, à la suite de Roman Jakobson, des gens s'intéressaient à l'histoire de l'art, mais à travers une forme de sémiologie structurale, où il s'agissait d'appliquer des schémas saussuriens et post-saussuriens à des objets non linguistiques. Un champ dans lequel le logos donnait un modèle théorique aux signes non linguistiques. Le texte de Schapiro reposait sur un autre modèle que celui de Jakobson, celui de Charles $\mathrm{S}$. Peirce, et il permettait de sortir de ce schéma.

- Atsushi Miura. Dans ce texte, «Sol », vous vous intéressez à la structure synchronique, plutôt qu'à la filiation diachronique. Peut-on y trouver un reflet du structuralisme de l'époque?

- Jean-Claude Lebensztejn. Probablement. Je suis resté assez fidèle à cela. J'ai écrit un autre texte sur le fauvisme et encore un sur l'expressionnisme, dans deux catalogues publiés par le musée d'Art moderne de la Ville de Paris il y a quelques années ${ }^{21}$, où je radicalise les positions de «Sol ». Je crois que l'on peut penser l'évolution de ce qu'on appelle les styles - un concept très impropre -, en termes d'évolutions structurelles. Il y a, à une époque donnée de l'histoire de l'art - dans ce cas, la fin du XIXe siècle et le début du XXe siècle - des schémas que l'on peut théoriser. II m'a semblé que l'on pouvait appeler « expressionnisme » toute la pensée d'avant-garde dans les arts visuels (mais pas seulement), qui joue sur la crise de la représentation, et qui comporte en peinture deux aspects presque contemporains l'un de l'autre, un expressionnisme de la couleur et un expressionnisme de la forme : ce qu'on appelle généralement le fauvisme, d'un côté, et le cubisme, de l'autre. Pour moi, ce ne sont pas des styles qui se suivent, mais des aspects qui ont une sorte de contemporanéité, avec une légère antériorité du fauvisme, qui tient à quelque chose dont parle Kant, à savoir que la couleur est moins ancrée dans la représentation que la forme. Il me semble qu'il est possible de penser le schéma de l'évolution des formes artistiques d'une manière un peu plus réfléchie et un peu plus globale qu'on ne le fait quand on parle de ces écoles.

J'irai plus loin. J'ai fait un petit texte sur Seurat et la science, paru dans Critique après la grande exposition Seurat à Paris et à New York ${ }^{22}$. C'est un double compte rendu, du catalogue de l'exposition, et de la réédition de l'Optique physiologique de Hermann von Helmholtz ${ }^{23}$, 
un ouvrage qui s'intitule « Manuel » en allemand (Handbuch...), mais qui est une somme gigantesque. J'ai relevé les manières très différentes dont les spécialistes de Seurat ont envisagé son rapport avec la science. Généralement, on écrit ou bien que Seurat a appliqué des données de la science contemporaine, celles de Michel-Eugène Chevreul et d'autres (comme le dit William Innes Homer), ou bien qu'il les a ignorées (comme le dit Richard Thomson), ou bien qu'il ne les a pas comprises (comme le dit Alan Lee), parce qu'on s'est quand même rendu compte qu'il y avait des différences. En essayant de penser ce rapport complexe de Seurat à la science, j'en suis arrivé à cette conclusion qu'il y a, non pas seulement chez Seurat, mais à son époque - les années 18801890 -, une pensée généralisée de ce que j'appelle le discontinu, et que l'on retrouve non seulement dans l'art, mais dans la poésie, les sciences du vivant, les sciences de la nature, la chimie et la physique, et dans d'autres objets tels que la linguistique. Et en mathématiques, la théorie de Cantor, contemporain de Seurat, est également une pensée du saut, du discontinu. Je fais une extrapolation peut-être abusive. Il y a un choix théorique ici. Une réflexion synchronique non seulement à l'intérieur de I'histoire de l'art, mais sur l'histoire de l'art en rapport avec l'histoire des sciences de la même époque, où des données entrent dans le même espace théorique généralisé sous ce nouveau mot d'ordre du discontinu. Dans ce texte, je suis allé plus loin dans l'élargissement de la conception synchronique.

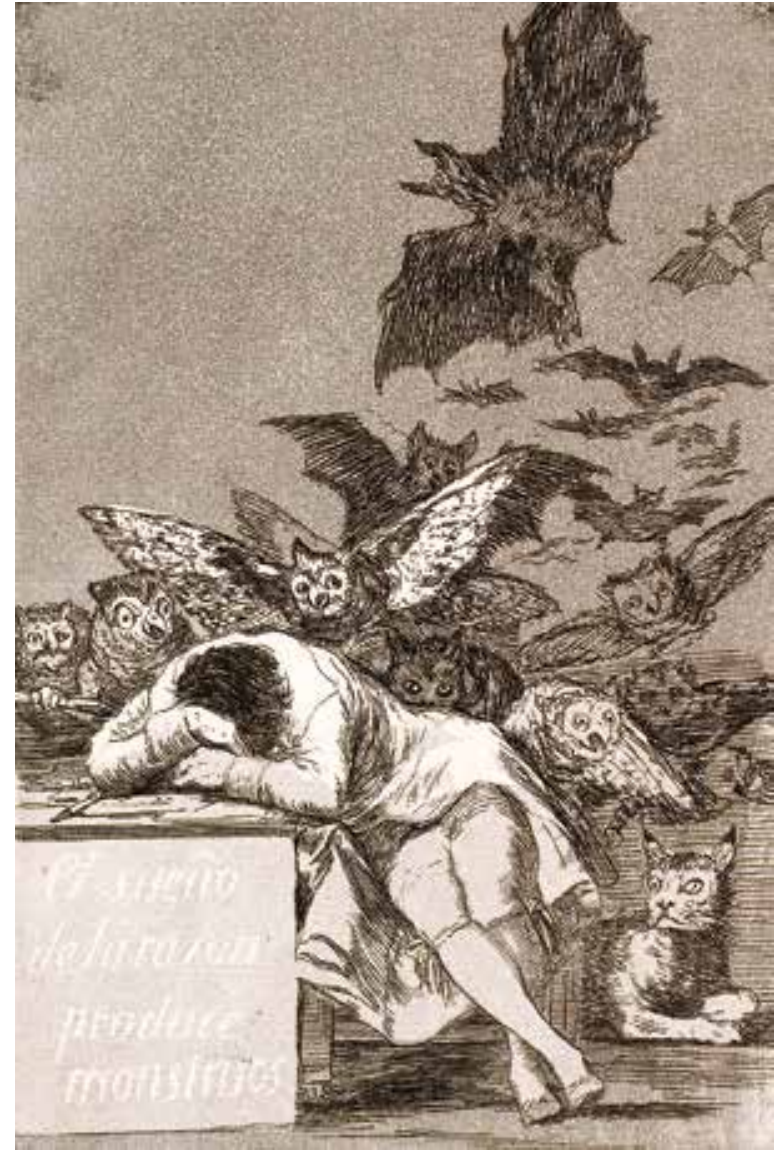

5. Francisco José de Goya y Lucientes, Le sommeil de la raison engendre des monstres ( $\left.n^{\circ} 43\right)$, Caprices, 1799, Kansas City (Missouri), The Nelson-Atkins Museum of Art, inv. 33-1077.

- Atsushi Miura. Je voudrais maintenant parler de «Au beauty parlour » et « Florilège de la nonchalance ", deux articles difficiles et stimulants ${ }^{24}$. Vous y traitez de la surface et de I'apparence : du problème de la couleur que vous comparez au maquillage et de celui de la touche en tant qu'elle relève de la sprezzatura. Là encore, je trouve une certaine "parergonalité " paradoxale, à la fois concrète et abstraite, qu'on pourrait approcher de cet idéal de l'« art qui dépasse l'art ", ou par laquelle la technè se dépasse... Voulez-vous expliquer un peu mieux votre point de vue?

- Jean-Claude Lebensztejn. J'ai trouvé là des éléments, je ne dirais pas paradoxaux, mais un peu dérangeants dans le domaine de la représentation. La couleur comme supplément et comme maquillage est une thématique que l'on rencontre assez fréquemment à partir du XVII siècle, surtout dans la discussion entre les poussinistes et les rubénistes à l'Académie de peinture, de sculpture et d'architecture. Un grand débat, dans lequel le coloris a été 
assimilé au maquillage ou au fard. Le coloris n'appartient pas, par conséquent, au corps de la peinture ; il est une sorte de supplément. Il est intéressant que les adversaires de la position académique de Charles Le Brun, qui déprécie la couleur au nom de cette opposition du corps et du fard, acceptent l'idée adverse de la couleur comme fard ; ainsi, Roger de Piles explique que « la Peinture n'est qu'un fard, qu'il est de son essence de tromper, \& que le plus grand trompeur en cet art est le plus grand Peintre ${ }^{25} »$.

- Atsushi Miura. Mais, dans «Au beauty parlour », vous ne développez pas tant l'argumentation sur le débat du coloris. II me semble que vous préférez plutôt passer à la problématique de la mort par l'intermédiaire des textes de Baudelaire et de Mallarmé.

- Jean-Claude Lebensztejn. Mon fil conducteur était ces mots de « fard » et de « maquillage», la manière dont ils réapparaissent et se modifient, disons, en gros, à l'époque de Manet. Dans le texte de Baudelaire, mais également dans ceux de Mallarmé et de Huysmans, il est dit - en retournant l'argumentation de l'époque antérieure - que le fard est l'essence de la peinture, rien d'autre. Cela reprend l'idée baroque de Roger de Piles, mais d'un nouveau point de vue, expliqué par Huysmans, à savoir que derrière du maquillage, il n'y a rien, sauf peut-être l'idée d'un corps en décomposition, tel qu'il est déjà représenté et thématisé dans

la peinture de Goya appelée Les Vieilles ou ¿Que tal? (Comment ça va ?), dans laquelle on voit des corps quasiment morts, qui se recouvrent de maquillage (fig. 6) : c'est presque une autoreprésentation

6. Francisco José de Goya y Lucientes, Les Vieilles, Le Temps ou ¿Que tal?, vers 1810, Lille, palais des Beaux-Arts, inv. P 50.

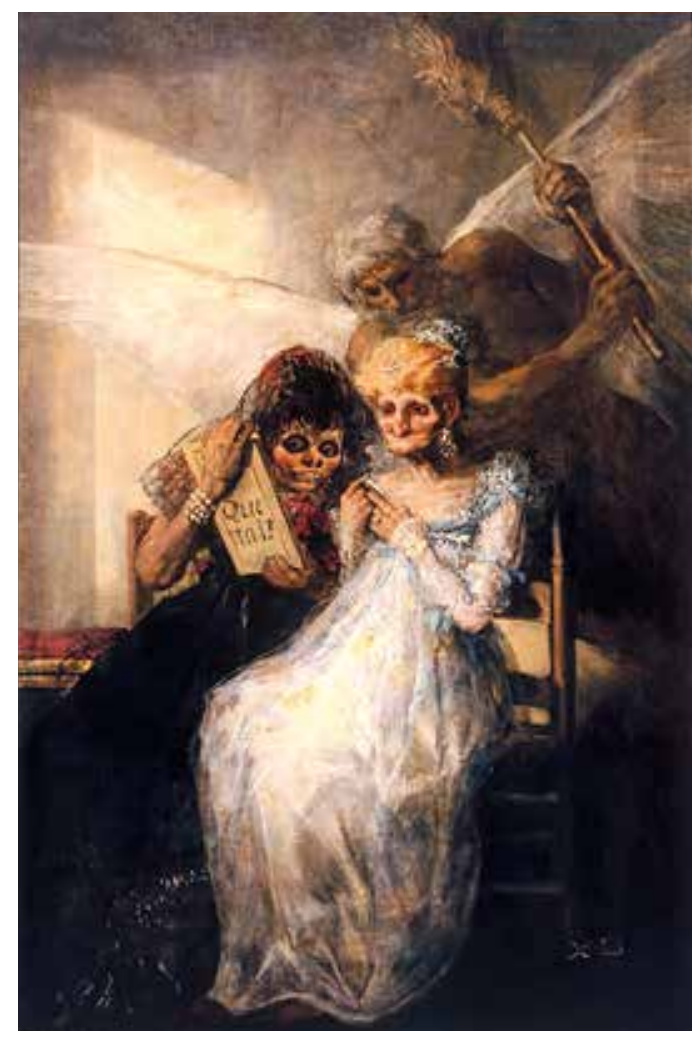
de la peinture telle que Goya peut l'envisager.

- Atsushi Miura. Et jusqu'à Andy Warhol...

- Jean-Claude Lebensztejn. Jusqu'à Andy Warhol.

- Atsushi Miura. Et dans « Florilège de la nonchalance ", vous analysez les problèmes de la tache et de l'esquisse en les mettant en rapport avec la notion de sprezzatura. Et ce terme italien me paraît sémantiquement très riche.

- Jean-Claude Lebensztejn. Je suis le fil du mot, comme pour le maquillage. Le terme de sprezzatura est polysémique, et même intraduisible. C'est un néologisme inventé par Baldassare Castiglione. Je cherchais des études sur le concept de sprezzatura, et j'ai été très étonné de ne rien trouver. Je me suis dit : si cela n'existe pas, je vais le faire. En suivant le fil de ce mot à travers la littérature italienne et espagnole, où il y a un concept un peu semblable, et dans l'espace, disons, classique qui est celui des $X V I^{\mathrm{e}}-\mathrm{XVII}{ }^{\mathrm{e}}$ siècles - entre Castiglione et Baltasar Gracián - j'ai trouvé qu'il y avait là quelque chose qui travaillait la représentation, un peu comme ces autres termes que vous avez évoqués, et qui 
permettait de remettre en question l'idée d'une représentation classique. Autrement dit, il n'y a pas simplement quelque chose qui serait l'essence de la peinture classique; cet espace est travaillé par ces termes parergonaux que sont le cadre et la signature, et la sprezzatura, sorte d'essence non essentielle. Comme le dit Roger de Piles dans un texte que je cite à la fin de l'article ${ }^{26}$, il est possible d'affirmer que la perfection de la peinture n'est pas son essence.

- Atsushi Miura. En lisant ce texte, je crois que l'on peut dire que vous avez fait dans I'histoire de l'art ce que Derrida a fait dans la philosophie.

- Jean-Claude Lebensztejn. Ce serait un peu présomptueux de répondre oui... Le champ de l'histoire de l'art est plus spécifique que celui de la philosophie. Mais il offre la possibilité d'une réflexion sur un mode de signifiance qui est celle de l'image, que la philosophie a généralement du mal à penser. S'interroger sur l'image comme la philosophie l'a fait sur le mot, le concept, le texte, etc., est un programme problématique et ambitieux, qui offre des possibilités intéressantes aujourd'hui, à une époque où la pensée de l'image paraît se développer, où se manifeste une sorte d'explosion, technologique et autre, des fonctions de l'image. Prendre l'image comme un objet de réflexion déconstructrice mérite considération.

- Atsushi Miura. II nous faut parler de vos autres ouvrages. Mais, puisque vous avez beaucoup écrit, je voudrais me borner au thème du parergon. D'abord, j'aimerais évoquer deux articles parus dans Zigzag ${ }^{27}$. Vous avez écrit "L'espace de l'art » et " L'étoile", sur Dubuffet et sur Stella. Ces deux articles concernent le musée et l'art, donc les institutions invisibles, ou encore le cadre au sens large du terme. Il s'agit toujours là du thème du parergon.

- Jean-Claude Lebensztejn. Oui, bien sûr. À propos du cadre, Derrida dans La Vérité en peinture renvoyait, en même temps qu'au texte sémiotique de Schapiro, à tout ce que j'avais publié28. Et en effet tout ce que j'avais écrit touchait d'une certaine manière à la question du cadre, de la limite, et par conséquent du parergon.

- Atsushi Miura. Vous avez écrit aussi « Les textes du peintre», sur Matisse. Je pense que cet artiste est toujours très important pour vous. Est-ce un peintre de référence parmi d'autres?

- Jean-Claude Lebensztejn. Tous les textes que j'ai écrits à cette époque sont des textes de circonstance. Dans le texte sur Matisse, la circonstance était double. D'une part, la grande et très importante exposition Matisse, en $1970^{29}$; d'autre part, la publication de ses textes, en français et en anglais ${ }^{30}$. J'ai fait un compte rendu de ces deux choses. II s'agissait de réexaminer la position de Matisse, qui, en France, était à ce moment-là l'objet d'une conception extrêmement réductrice. L'exposition réalisée par Pierre Schneider et l'édition des écrits de Matisse par Dominique Fourcade ont permis de la reconsidérer. Ce texte est donc lié à cette actualité ; je m'intéresse toujours beaucoup à Matisse, et j'ai donné en 1987 et 1991 deux conférences, publiées plus tard, sur Matisse et l'espace, et sur Matisse à Tanger ${ }^{31}$. Mais ce n'est plus pour moi un objet de réflexion dans la mesure où ces choses-là ont été assimilées et sont même devenues une sorte de dogme. Je me suis penché de nouveau sur Matisse dans le cadre de ce deuxième article sur le fauvisme, publié en 1999 à l'occasion de l'exposition sur le fauvisme en Europe ${ }^{32}$.

7. Stéphane Mallarmé, Un coup de Dés jamais n'abolira le Hasard, 1897, p. $8 \mathrm{v}^{\circ}-9 \mathrm{r}^{\circ}$, tirées d'un jeu d'épreuves de l'édition projetée par Mallarmé, dans la mise en page conçue par lui et restée inédite. Paris, BnF, réserve des Livres rares, inv. RESFOL-NFY-130. 
soucienx

expiatoire et pubere muet

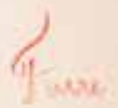

La lucide et seigneuriale aigrette au front imvisible scintille puis ombrage une stature mignonne ténébreuse en sa torsion de sirène

por dimpatientes squanes ultimes 
rive

que

SI

de vertige

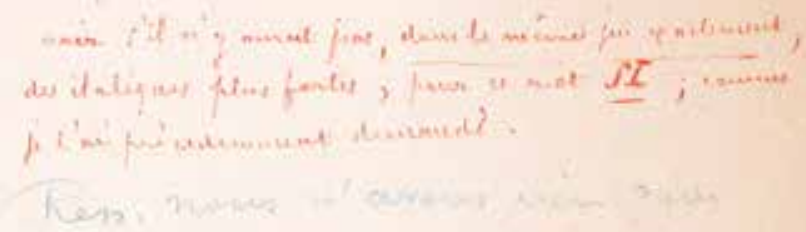

debout

bifurquées souffleter $\mathrm{fl}$ (f)

un roc

faux manoir
tout de suite

évaporé en brumes

qui imposa

une borne à Pinfint

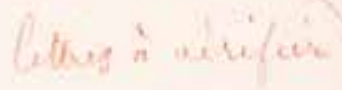


Mais je me suis intéressé aussi à d'autres artistes de cette période, comme Piet Mondrian. Je me suis occupé aussi de certains artistes contemporains, Brice Marden, Malcolm Morley. Il s'agissait de commandes de circonstance.

- Atsushi Miura. Quant à Zigzag en tant que livre, il me semble que c'est un ouvrage à la fois mallarméen et derridien.

- Jean-Claude Lebensztejn. II ne l'a pas été a priori. II s'agissait simplement de reconsidérer ce que c'est qu'un livre, et l'espace du volume, le volume du volume : les deux dimensions de la page, mais également la troisième dimension des feuilles qui se superposent. C'est pourquoi le titre parcourt tout le livre, de manière à mettre en évidence le caractère volumétrique du volume.

- Atsushi Miura. Comment avez-vous conçu cette forme?

- Jean-Claude Lebensztejn. En m’attachant à la matérialité du livre. La simple considération matérielle du livre - la pensée que la matérialité du livre fait partie du texte - a été le point de départ de ma réflexion. J'ai repensé à ce que dit Mallarmé, dans son texte sur le Livre : " admis le volume qui ne comporte aucun signataire, considérer quel il est ${ }^{33}$ ». J'ai fait un compte rendu des éditions d'Un coup de Dés ${ }^{34}$, où je parle de la conception typographique que Mallarmé avait du livre (fig. 7). Dans une lettre, il se présente comme "le lettré qui mise à ce que le texte fasse corps avec le papier même ${ }^{35}$ ». L'opposition traditionnelle, et je dirais, chrétienne, cette opposition entre le contenu du texte et la matérialité du livre peut être déconstruite, comme le fait Mallarmé écrivant Un coup de Dés.

- Atsushi Miura. Je voudrais évoquer ensuite votre ouvrage monumental sur Alexander Cozens.

- Jean-Claude Lebensztejn. Ce n'est qu'une thèse.

- Atsushi Miura. Je ne peux pas résumer ce livre ${ }^{36}$. Mais, par rapport à la problématique du parergon, je voudrais dire ceci : vous avez choisi le traité d'Alexander Cozens, un texte parergonal par un personnage périphérique. Mais vous en avez tiré les problématiques centrales, voire essentielles, relatives à l'histoire de l'art occidental. C'est très impressionnant.

- Jean-Claude Lebensztejn. On y reviendra plus loin, mais ma méthode est précisément là. Je m'attache à des détails qui font problème, qui sont apparemment extérieurs au champ dans lequel ils se trouvent. Par exemple Cozens : il a écrit ce petit traité, Nouvelle méthode pour secourir l'invention dans le dessin des compositions originales de paysage, en 1785, avec des taches quasi abstraites, dont on a dit qu'elles annonçaient l'art abstrait (fig. 8). En lisant le texte, je me suis rendu compte que Cozens traversait tout le domaine de l'esthétique de son temps, l'époque classique, et plus précisément néoclassique. Ce qui m'intéressait, c'était de savoir comment des données esthétiques tout à fait courantes, les lieux communs de son temps, ont pu aboutir à ces images. Toutes les fois que je me suis interrogé sur quelque chose, je l'ai fait par le biais de détails intrigants, un peu à la façon d'un détective qui voit un objet qui ne colle pas avec les données de l'histoire, et qui va chercher la logique qui permettra soit de changer l'histoire, soit de réintégrer dans l'histoire l'objet en question. 


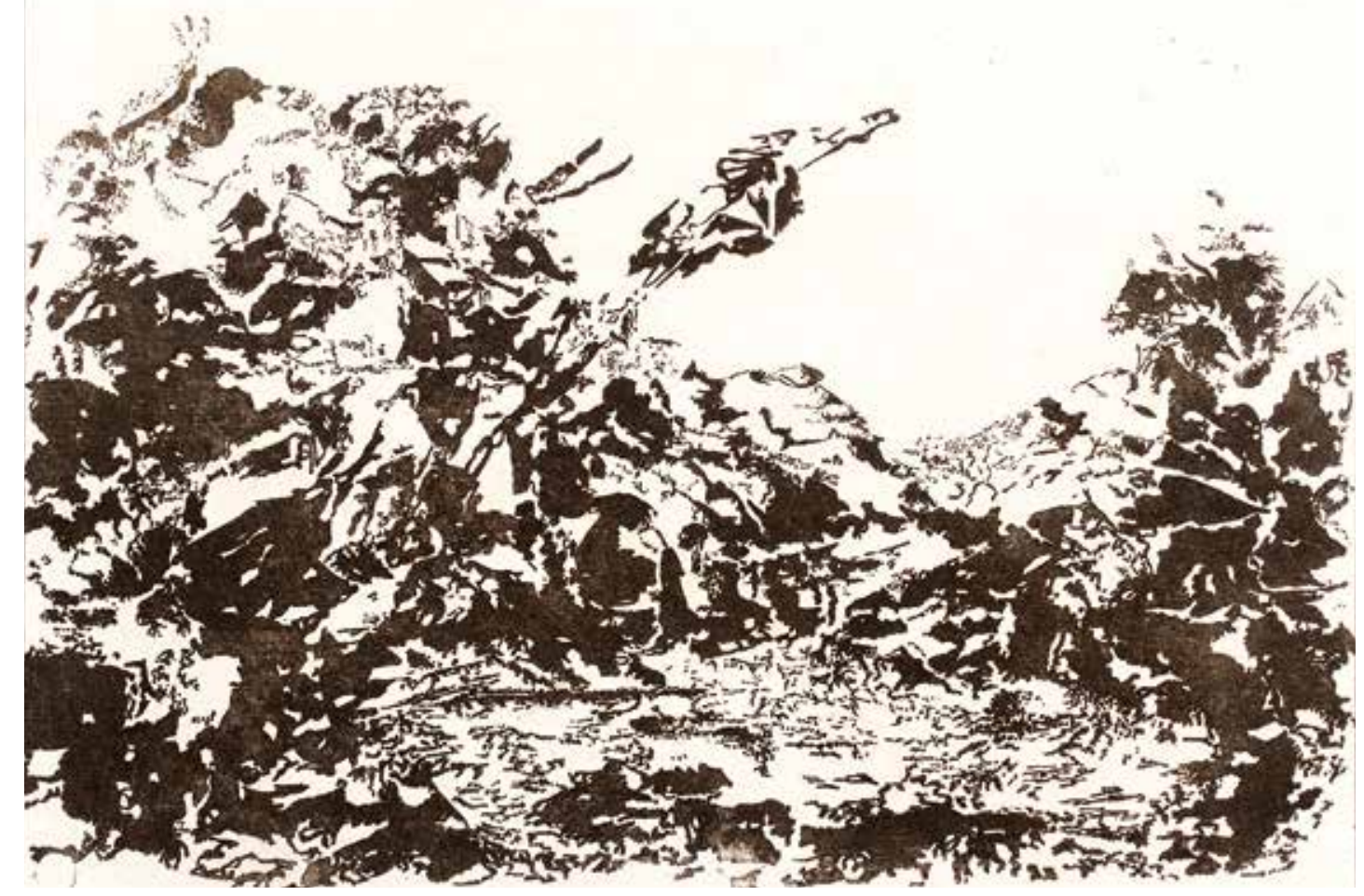

- Atsushi Miura. Vous y avez mentionné Théorie du nuage $d^{\prime}$ Hubert Damisch. Quelle est votre relation avec Damisch?

8. Alexander Cozens, A New Method of Assisting the Invention in Drawing Original Compositions of Landscape, Londres, 1785, pl. 12, Londres, Tate,

- Jean-Claude Lebensztejn. J'ai assisté à ses premiers sémiinv. T03180. naires, à la fin des années 1960 et au début des années 1970. J'ai mentionné son livre sur le nuage, parce qu'il y avait là des problèmes qui recoupaient la question de la tache. À ce moment-là, j'ai beaucoup fait référence à certains historiens de l'art classiques, comme Robert Rosenblum, Hugh Honour, et d'autres qui se sont intéressés à l'époque de Cozens, en les utilisant à ma manière, suivant les intentions qui étaient les miennes. Et j'ai fait une utilisation classique de Damisch, pas tellement de sa problématique théorique, mais plutôt des données historiographiques qu'il pouvait apporter par l'étude des nuages, et des images dans les nuages. Tout cela est restreint au chapitre sur « La double origine de la tache ».

- Atsushi Miura. Le XVIII siècle, c'est l'époque où l'on peut trouver une sorte de fusion entre les éléments classiques et anticlassiques.

- Jean-Claude Lebensztejn. II me semble que la question du classique et de l'anticlassique est une manière un peu brutale de poser les choses. Je les poserais plutôt en termes systématiques à travers ces couples d'opposition, néoclassique / préromantique, beau / sublime, Sud / Nord, Apollon / Dionysos, ou dessin fini / esquisse, qu'il faut envisager dans un ensemble systématique, non pas dans une opposition diachronique ou dualiste. 
JEAN-CLAUDE

LEBENSZTEJN

CHAHUT

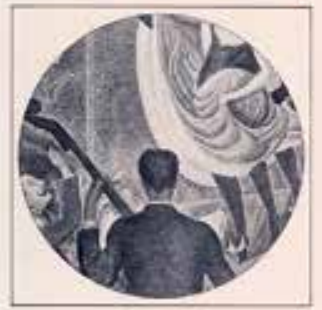

muax

asicten ist

9. Jean-Claude Lebensztejn, Chahut, Paris, Hazan, 1989.
- Atsushi Miura. Vous n'avez pas comparé le paysage de Cozens avec le paysage oriental.

- Jean-Claude Lebensztejn. Cela ne fait pas partie de ma culture. Pour toucher à cette question, il faudrait connaître vraiment le chinois, non seulement la langue chinoise mais également les divers modes de pensée qui traitent du paysage. J'en ai un peu parlé, parce qu'on a vite fait le rapprochement avec le paysage chinois, dès qu'on s'est intéressé à Cozens au début du XXe siècle. Mais ce domaine est trop étranger à ma culture, bien que je m'intéresse beaucoup à la pensée taoïste et à l'art chinois en général, surtout aux arts de la période Song. Il est très difficile de penser des espaces culturels aussi différents, en se basant uniquement sur des données de ressemblance formelle. Ce serait un grave danger. Je préférais l'éviter plutôt que de faire des rapprochements douteux et superficiels.

- Atsushi Miura. Vous avez écrit sur Seurat (fig. 9). Je crois qu'il est I'un de vos peintres de prédilection. Votre Seurat est déroutant, solitaire, toujours à côté de l'histoire de l'art « normale $»^{37}$.

- Jean-Claude Lebensztejn. Tous les peintres qui m'intéressent sont des peintres déroutants et solitaires. Je pense à Pontormo, qui m'a beaucoup retenu à un moment, et qui est, par certains côtés, très proche de Seurat. Mais comme je le disais, ce sont des figures, des détails, ou des artistes qui sont en quelque sorte décalés ou déroutants, qui me permettent d'envisager et de reconsidérer le rapport entre la marge et la centralité, de repenser l'idée de centre, et même de la déporter, de telle manière que le centre n'est plus tellement ce que l'on pensait qu'il était, mais que le centre et la marge jouent et s'intriquent comme dans l'espace de la page mallarméenne. Mallarmé écrit, dans un texte d'introduction qu'il a fait pour la première publication d'Un coup de Dés, que, dans un poème classique, le texte " occupe, au milieu, le tiers environ du feuillet ». Et il ajoute : « je ne transgresse cette mesure, seulement la disperse ». En reportant le blanc, qui d'habitude entoure le texte, au milieu du texte, il déplace l'idée de marge et de centre. Le principe d'une marginalisation de la marge et d'une centralité du centre est défait par le texte de Mallarmé. C'est quelque chose qui est à la fois physique et en même temps abstrait, conceptuel. C'est pourquoi mes livres se présentent comme ils le font, mais également de manière à mettre en rapport cette décentralisation du centre avec des figures qu'on peut qualifier de déroutantes.

\section{- Atsushi Miura. C'est très intéressant. Quel est votre intérêt pour Pontormo ?}

- Jean-Claude Lebensztejn. L'intérêt pour Pontormo est venu, au départ, d'un texte tout à fait mineur : les quelques pages de son journal où il décrit ce qu'il a mangé et ce qu'il a fait, etc. (fig. 10). J'ai été très impressionné et troublé par cette lecture. Il fallait que je règle ce problème, non pas simplement avec le texte, mais avec moi-même. Je l'ai réglé en écrivant. C'est un travail qui m'a pris trois ans, et qui est peut-être ce que j'ai fait de plus radical, entre autres eu égard à cette méthodologie consistant à donner une telle importance à un texte apparemment privé de toute forme d'intérêt. C'est un peu la même méthode que dans Cozens, 
mais poussée plus loin. Et la forme n'est plus la forme de la thèse. Dans Cozens, j'ai décidé de jouer le jeu classique de l'écriture de thèse, qui pose objectivement des choses, avec les notes en bas de page, la bibliographie, l'index, tout cela. Dans Pontormo, j'implique ma propre personne de manière plus profonde. L'écriture est beaucoup plus troublée, mêlée et moins objective, d'apparence beaucoup moins scientifique. Mais je pense que l'idée même d'apparence scientifique est une idée à revoir.

- Atsushi Miura. De là on passe au problème de la méthodologie. J'ai peur de me tromper, mais il me semble que vous avez été pionnier dans un courant actuel de la nouvelle méthodologie de l'histoire de l'art (ou bien de l'analyse de l'image), représenté par exemple par Victor Stoichita, qui traite du problème de la méta-peinture et de celui de l'ombre, et par Daniel Arasse qui traite des œuvres du point de vue de détails, et aussi par Georges Didi-Huberman et son concept de l'anachronisme. Tous ces chercheurs traitent d'une certaine manière du problème du parergon.

- Jean-Claude Lebensztejn. D'une certaine manière, on peut dire cela. Mais ce ne sont pas eux que je fréquente dans ma discipline. J'ai lu plusieurs textes des uns et des autres, mais ce ne sont pas des textes qui m'ont beaucoup inspiré personnellement. Mon inspiration, vous la retrouvez ailleurs. Je cite mes sources, il est donc facile de voir ce qui m'a inspiré. Ce ne sont pas forcément des gens qui travaillent de la même manière que moi.

\section{- Atsushi Miura. Quelle est la différence?}

- Jean-Claude Lebensztejn. Je ne sais pas, chacun a sa spécialité... ! Arasse travaille dans un domaine de la représentation classique. Il lui arrive par exception de parler de l'art contemporain. Stoichita s'est intéressé au journal de Pontormo. Il a fait une traduction roumaine de ce texte avec une préface en roumain.

- Atsushi Miura. Est-il alors plus proche de vous?

- Jean-Claude Lebensztejn. Non, je ne le pense pas. Si je peux dire une chose un peu brutale, je suis par formation philologue, et lui ne l'est pas. Son édition de Pontormo repose sur une erreur, qui n'est pas la sienne : l'édition Cecchi qu'il a traduite a bouleversé l'ordre chronologique et, par conséquent, la signification du journal ${ }^{38}$. J'ai parlé de cette édition dans le commentaire que j'ai fait du journal de Pontormo ${ }^{39}$. Parce qu'il a traduit à partir de ce texte, qu'il l'a donc regardé de près, Stoichita aurait pu s'en rendre compte. Mais il ne l'a pas fait. II n'a pas eu ce réflexe philologique de se dire : ici, quelque chose ne va pas. Stoichita

10. Jacopo da Pontormo, Journal, Florence, Biblioteca Nazionale Centrale, Magl. VIII 1490, fol. $67 \mathrm{r}^{\circ}$

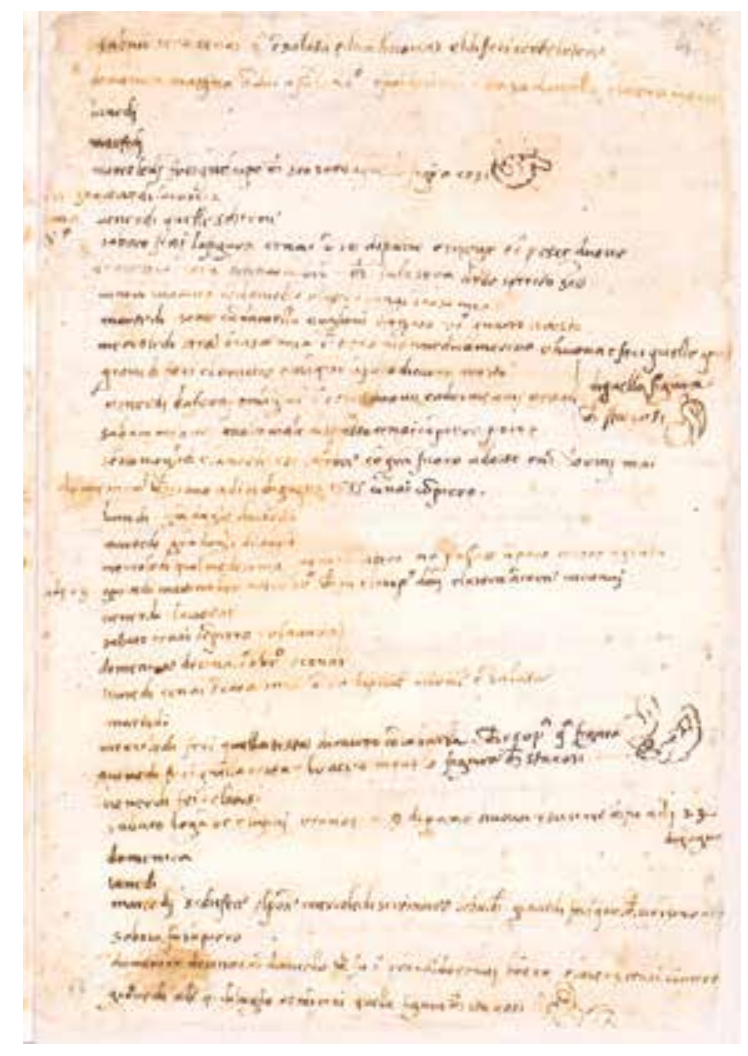


s'est fié à cette édition ; en travaillant sur la même édition, je me suis rendu compte que quelque chose clochait, et je me suis dit : il faut absolument voir la source, le manuscrit du journal. Et en regardant le manuscrit, je me suis rendu compte tout de suite de ce qui n'allait pas. Ce qui m’a stimulé n'était pas seulement le caractère accessoire, inessentiel du journal, mais le fait que, dans cette édition courante du journal, quelque chose n'allait pas.

Je dirais donc que la différence générale est celle-ci. Nous sommes tous dans un espace de délire. Mais, dans mon cas, le délire repose sur des bases très précises. Un autre exemple. Dans Le Détail, Arasse fait de longs commentaires sur une ombre dans un tableau d'Ingres, où je ne vois pas ce qu'il voit. Il a le droit de voir ce qu'il voit, mais je ne vois pas ce qu'il voit ! Cela ne dit pas que mon regard est meilleur que le sien, ni que le sien est meilleur que le mien. Ce sont des regards différents. Pourquoi sont-ils différents ? Je ne sais pas. Pour moi, c'est beaucoup plus simple que ce qu'il en dit. Arasse est, comme moi, normalien et littéraire. Il a une formation que, je crois, Stoichita n'a pas, et que Didi-Huberman n'a pas non plus, une formation textuelle, qui lui permet de s'attacher à l'image avec un peu plus de sérieux. Mais - je parle du Détail - il y a là quelque chose qui est de l'ordre du fantasme. Et le fantasme de l'un n'est pas le fantasme de l'autre. Je pense que ce qui réunit ces quatre noms que vous avez prononcés, c'est peut-être la pensée d'une histoire de l'art qui intègre le fantasme dans la réflexion. Mais chacun a ses fantasmes, et tous les fantasmes sont respectables, pourvu qu'ils se donnent pour fantasmes. Et je crois que si l'on accepte le fantasmatique dans le champ de la réflexion, on peut aboutir à de grandes choses.

De ce point de vue, Warburg est la figure incontournable. Il est un peu comme le Saussure des Anagrammes : quelqu'un chez qui on ne sait jamais si ce qu'il pense est de l'ordre de la construction illusoire ou si cela recouvre ou découvre une réalité. Il y a une problématique du rapport entre le fantasme, la construction mentale et la réalité, qui reste un indécidable.

Ma méthode spécifique, c'est de prendre un objet concret et singulier que j'examine dans toute sa rigueur. Et qui sera mon point de départ pour une réflexion dans laquelle j'utilise toutes les données, théoriques, concrètes, historiques, méthodologiques, visionnaires, fantasmatiques, que je tâche d'intégrer dans le texte, et par lesquelles j'essaie d'innerver le texte. Cela m'oppose à certaines procédures à l'américaine (de Rosalind Krauss et d'autres), dans lesquelles on commence par construire une grille théorique que l'on essaie d'appliquer à un objet. Ma méthode est inductive. Elle part d'un objet concret et particulier, et essaie $d^{\prime}$ irradier à partir de là.

- Atsushi Miura. Dans la méthodologie de l'histoire de l'art anglo-saxonne, je perçois en effet de temps en temps un certain réductionnisme où l'on est tenté d'expliquer une œuvre par des références externes.

- Jean-Claude Lebensztejn. J'ai fait une critique méthodologique d'un travail féministe $\mathrm{d}^{\prime}$ Ewa Lajer-Burcharth sur David ${ }^{40}$. Vous trouverez là des propositions méthodologiques qui se terminent par cette conclusion un peu ambitieuse, que l'histoire de l'art reste à faire.

- Atsushi Miura. La dernière question concerne un thème peut-être inattendu : le Japon, ce pays d'Extrême-Orient que j'oserais qualifier de quelque peu annexé, par rapport au continent. Je trouve de temps en temps des éléments japonais dans vos ouvrages.

- Jean-Claude Lebensztejn. Ah bon?

- Atsushi Miura. Vous citez par exemple un propos de Hokusai au début de Zigzag. 


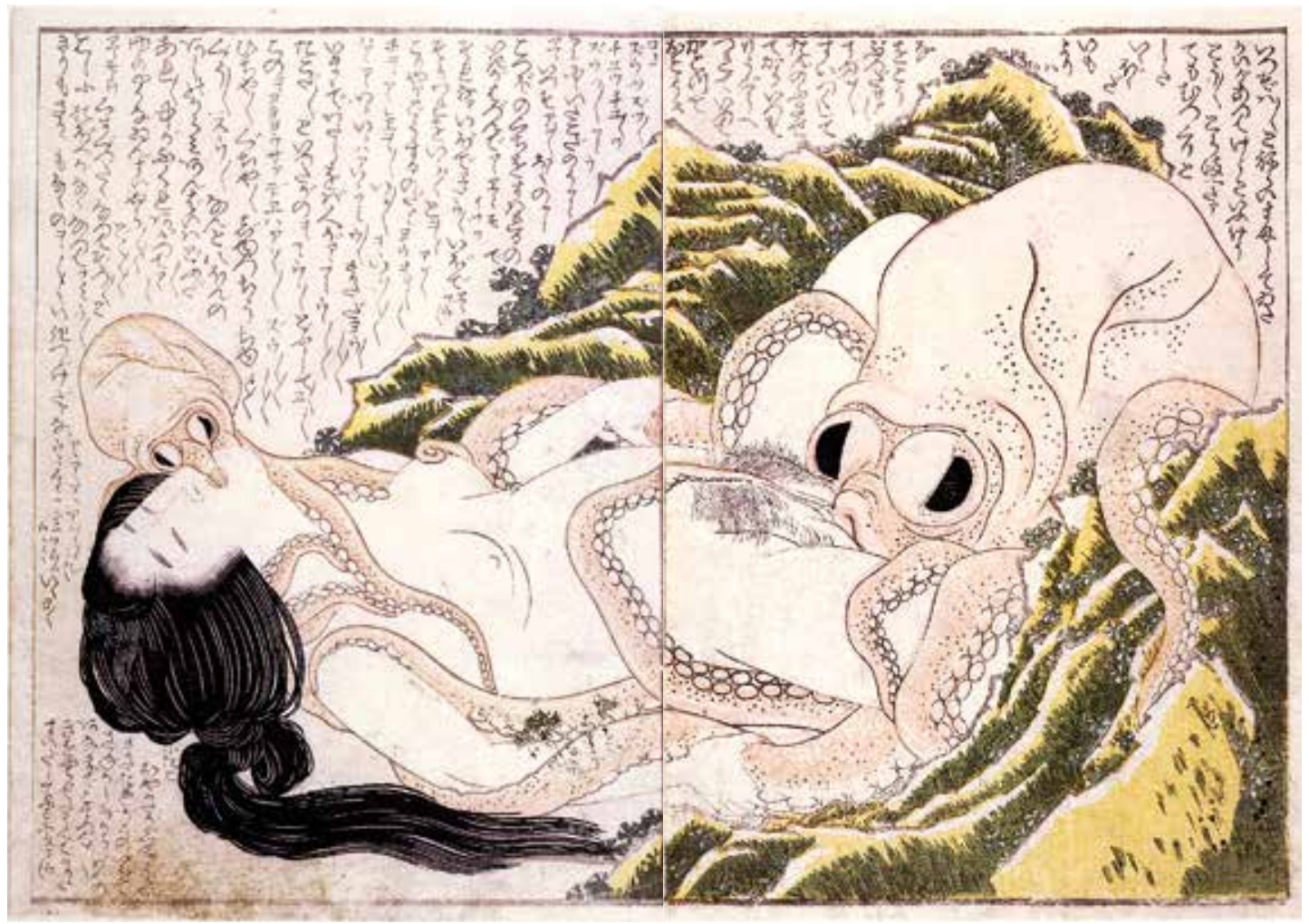

- Jean-Claude Lebensztejn. Je ne suis pas le seul. Depuis Edmond de Goncourt, on l'a cité des milliers de fois ! Ce propos de Hokusai m'intéresse beaucoup ; il affirme que ce n'est que dans la mort que le dessin devient vivant: quand

11. Katsushika Hokusai, Le Rêve de la femme du pêcheur, publié en ouverture de Kinoe no komatsu 喜能会之故真通, 1814, Londres, The British Museum, inv. OA+,0.109. j'aurai l'âge de cent dix ans, dit-il, chaque point, chaque ligne seront vivants. Il prévoyait sans doute qu'à ce moment-là il serait mort. C'est ce paradoxe qui m'intéressait.

Je ne connais pas bien l'art japonais. J'ai entendu dire de la part de Japonais que Hokusai (fig. 11) n'était pas tellement apprécié au Japon, peut-être parce qu'il était trop occidental, trop mineur par rapport à l'art canonique japonais. Mais ce qui m'intéresse dans l'art japonais, ce sont des formes mineures. Je ne suis pas tellement touché par les grandes statues. La chose qui m'excite le plus dans ce Bouddha monumental de Nara, c'est que tous les ans, le 7 août, on en retire dix seaux de poussière. Je m'intéresse plutôt à des formes de l'art japonais qui sont considérées en Occident comme mineures, l'art des jardins, l'art du décor, l'art du costume, les stylistes contemporains. Beaucoup plus qu'aux formes classiques de la peinture, de la sculpture et de l'architecture.

- Atsushi Miura. Vous trouvez donc le singulier, dans l'art japonais, dans ce qui n'est pas classique, ni monumental, peut-être dans ses formes mineures?

- Jean-Claude Lebensztejn. Non, je ne l'appelle pas mineur. Pour moi, le bébé japonais, le jardin japonais, les cérémonies du thé, la manière de faire un paquet, la manière dont on noue une algue dans le bouillon, sont des formes d'art majeures. Par rapport aux normes occidentales, 


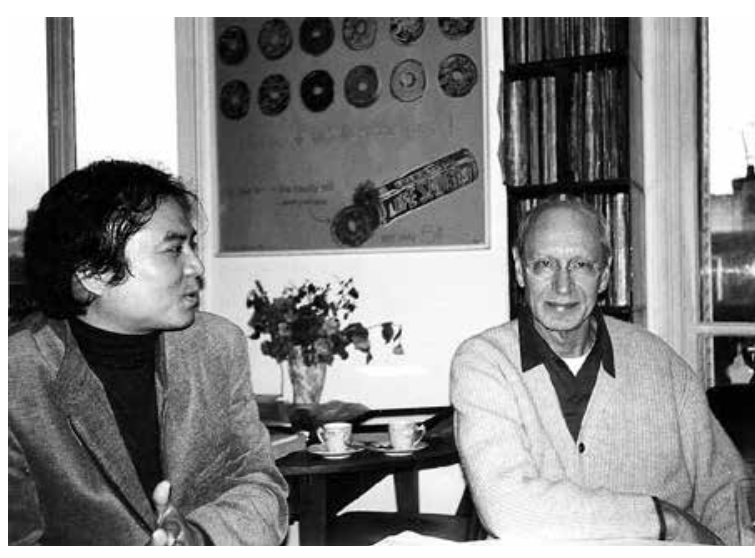

12. Jean-Claude Lebensztejn et Atsushi Miura, le 21 décembre 2002, à Paris. Archives de Sangensha, éditeur de la version japonaise de cet entretien, parue en 2003. il est clair que les grands Bouddha, les grands temples ou même la peinture classique ou contemporaine sont plus importants. Mais ce qui m'intéresse, ce sont ces autres formes d'art que j’ai vues du Japon.

- Atsushi Miura. Et vous évoquez quelques textes concernant le Japon dans Miaulique.

- Jean-Claude Lebensztejn. Oui. Il s'agit d'un petit livre du jésuite Luís Fróis, écrit en portugais en $1585^{41}$. Il est extrêmement drôle. Vous trouvez par exemple ce passage où il dit : en Europe, on salue en ôtant son chapeau ; au Japon, en retirant ses chaussures. Tout se fait à l'envers. II s'agit de formes oppositionnelles dans lesquelles la culture du Japon se trouve littéralement aux antipodes de la culture européenne, de manière très dualiste, mais totalement satirique et comique. Il y a évidemment un point de vue européocentriste, celui d'un jésuite qui va au Japon pour christianiser les populations locales, et qui trouve qu'ils sont de race inférieure. Mais il se rend compte aussi que les Japonais trouvent que les Européens sont de race inférieure. C'est un point de vue très intéressant. Et, en même temps, il y a des nuances. Les musiciens occidentaux répètent que la musique japonaise est affreuse, qu'elle est un miaulement. Mais si les Japonais ne peuvent pas, au $\mathrm{XVl}^{\mathrm{e}}$ siècle, entendre avec intérêt la musique européenne, ils sont beaucoup plus réservés, moins méprisants.

- Atsushi Miura. Comment pourrait-on réécrire l'histoire de l'art japonais du point de vue du parergon, même si, bien sûr, c'est notre propre problème?

- Jean-Claude Lebensztejn. II me semble que la chance de l'art japonais n'est pas forcément dans les formes d'art occidentalisées, mais dans d'autres formes considérées comme mineures, mais qui, à mon avis, sont les grandes forces de l'art japonais : la manière dont la nature est complètement retravaillée dans un jardin, par les greffes, les torsions de branches. Je pense que le génie de l'art japonais se trouve dans l'ingéniosité du détail, qui repense ou déjoue l'opposition de la nature et de l'artifice ${ }^{42}$. 


\section{NOTES}

1. Jean-Claude Lebensztejn, Imiter sans fin. Le chant de l'aimable Angelette, Montélimar, éditions du Limon, 1987, p. 27.

2. Jean-Claude Lebensztejn, Zigzag, Paris, Aubier Flammarion, 1980, p. 13

3. Jean-Claude Lebensztejn, Déplacements, Bruxelles, Les Presses du réel (coll. "Fabula »), 2013.

4. Jean-Claude Lebensztejn, Miaulique, Paris / New York, Le Passage, 2002, p. 91.

5. Son article sur Matisse ("Les textes du peintre», repris dans Lebensztejn, 1980, cité n. 2, p. 161-206) fut déjà traduit en 1981 ; mais encore avant, en 1976, celui - le premier texte de lui - sur « Un tableau de Titien / un essai de Panofsky » (dans Critique, $\mathrm{n}^{\circ} 315$ 316, 1973, p. 821-842 - je dois cette précision à l'auteur lui-même!).

6.『セザンヌのエチュード』浅野春男訳、三元社、1999 年。『猫の音楽』森元庸介訳、勁草書房、2014年。

7. Lebensztejn, 2002, cité n. 4, p. 63-87.

8. Jean-Claude Lebensztejn, "Florilège de la nonchalance ", dans Annexes - de l'œuvre d'art, Bruxelles, La Part de l'CEil, 1999, p. 227.

9. Lebensztejn, 2002, cité n. 4, p. 91.

10. L'entretien, transcrit et traduit par Yosuke Morimoto en 2002, a initialement été publié en japonais dans la revue 『西洋美術史研究』 [Études sur l'histoire de l'art occidental], n० 9 (numéro spécial sur le parergon), en 2003. Meyer Schapiro, "Leonardo and Freud: an Art-Historical Study ", dans Journal of the History of Ideas, $n^{\circ} 17$, 1956, p. 147-178.

11. Meyer Schapiro, "On some Problems in the Semiotics of Visual Art: Field and Vehicle in Image-Signs ", dans Semiotica, no 1, 1969, p. 223-242 ; Meyer Schapiro, "Sur quelques problèmes de sémiotique de l'art visuel: champ et véhicule dans les signes iconiques ", dans Critique, nos 315-316, août-septembre 1973, p. 843-866.

12. Meyer Schapiro, «Sur quelques problèmes de sémiotique de l'art visuel : champ et véhicule dans les signes iconiques ", "Léonard et Freud ", "Seurat ", dans Style, artiste et société, Paris, Gallimard, 1982.

13. Meyer Schapiro, "The Still Life as a Personal Object. A Note on Heidegger and Van Gogh ", dans Marianne L. Simmel (dir.), The Reach of Mind: Essays in Memory of Kurt Goldstein, New York, Springer Publishing Company, Inc., 1968.

14. Jacques Derrida, La Vérité en peinture, Paris, Flammarion (coll. «Champs »), 1978, p. 14.

15. Jean-Claude Lebensztejn, Annexes - de l'œuvre d'art, Bruxelles, La Part de l'CEil, 1999, p. 5.

16. Lettre du 28 avril 1639 à Chantelou, publiée dans Nicolas Poussin, Correspondance de Nicolas Poussin publiée d'après les originaux, Charles Jouanny (éd.), Paris, J. Schemit, 1911, p. 21.

17. Lucrèce, De la nature, livre IV ; sur Démocrite et Épicure, voir Plutarque, Propos de table, VIII, 10, 2.

18. Jean-Claude Lebensztejn, "Sans titre (océanique) », dans Brice Marden, Paris, Galerie Montenay, 1987, p. 7-15.
19. Michel Hoog et Leopold Reidemeister, Le Fauvisme français et les débuts de l'expressionnisme allemand, cat. exp. (Paris, Musée national d'art moderne / Munich, Haus der Kunst, 1966), Paris / Munich, Musée national d'art moderne / Haus der Kunst, 1966.

20. Schapiro, 1969, cité n. 11.

21. Jean-Claude Lebensztejn, "Tournant", dans Le Fauvisme ou l'épreuve du feu : éruption de la modernité en Europe, cat. exp. (Paris, musée d'Art moderne de la Ville de Paris, 1999-2000), Paris, Paris-Musées, 1999 ; "Douane-Zoll », dans Figures du moderne : l'expressionnisme en Allemagne, 1905-1914, Dresde, Munich, Berlin, cat. exp. (Paris, musée d'art moderne de la Ville de Paris, 1992-1993), Paris, Paris-Musées, 1992.

22. Jean-Claude Lebensztejn, "L'optique du peintre (Seurat avec Helmholtz) », dans Critique, mai 1992, t. XLVIII, no 540, p. 404-419. Voir Robert L. Herbert, Georges Seurat, 1859-1891, cat. exp. (Paris, Galeries nationales du Grand Palais, 1991 / New York, The Metropolitan Museum of Art, 1991-1992), New York, The Metropolitan Museum of Art, 1991.

23. Hermann von Helmholtz, Optique physiologique, Paris, Éditions Jacques Gabay, 1989 (fac-similé de la traduction française par É. Javal et Th. N. Klein, Paris, V. Masson et fils, 1867 [éd. orig. : Handbuch der physiologischen Optik, premièrement parue de 1856 à 1866 dans I'Encyclopédie physique de Gustav Karsten, puis sous la forme d'un volume séparé en 1867]).

24. Jean-Claude Lebensztejn, "Au beauty parlour", dans Traverses, $n^{\circ}$ 7, février 1977, p. 74-94; " Florilège de la nonchalance ", dans Critique, $\mathrm{n}^{\circ} 473$, octobre 1986, p. 1025-1052.

25. Roger de Piles, Dialogue sur le coloris, Paris, Langlois, 1673, p. 67, réédité en fac-similé : Genève, Minkoff, 1973.

26. Voir la note 24 ; Roger de Piles, "L'Idée du Peintre parfait ", dans Abregé de la vie des Peintres..., Paris, chez François Muguet, 1699, p. 27.

27. Jean-Claude Lebensztejn, Zigzag, Paris, Flammarion (coll. "La philosophie en effet »), 1981.

28. Derrida, 1978, cité n. 14, p. 90, note 1 .

29. Pierre Schneider, avec la collaboration de Tamara Préaud, Henri Matisse : exposition du centenaire, cat. exp. (Paris, Grand Palais, 1970), Paris, Réunion des musées nationaux, 1970.

30. Henri Matisse, Écrits et propos sur l'art, Dominique Fourcade (éd.), Paris, Hermann, 1972 ; Jack D. Flam, Matisse on Art, Londres, Phaidon, 1973.

31. Jean-Claude Lebensztejn, "Matisse et l'espace », dans Matisse aujourd'hui, Cahiers Henri Matisse, n 5, Nice, musée Matisse, 1993 ; " Matisse à Tanger », dans Tanger, Espace imaginaire, Tanger / Rabat, 1992, republié dans Le magazine, Centre Pompidou, nº 74, mars-mai 1993.

32. Lebensztejn, 1999, cité n. 21.

33. Stéphane Mallarmé, "Le Livre, Instrument Spirituel », $1^{\text {ère }}$ version, parue dans La Revue Blanche, juillet 1895, p. 33.

34. "Note relative au Coup de Dés ", dans Critique, juin-juillet, 1980, n 397-398, p. 633-659. 
35. Lettre à Edmond Deman, du 28 avril 1888, dans Stéphane Mallarmé, Correspondance : 1886-1889, Lloyd James Austin et Henri Mondor (éd.), Paris, Gallimard (" collection Blanche »), t. III, p. 188.

36. Jean-Claude Lebensztejn, L'art de la tache. Introduction à la "Nouvelle méthode" d'Alexander Cozens, Montélimar, éditions du limon, 1990.

37. Jean-Claude Lebensztejn, Chahut, Paris, Hazan, 1989.

38. Jacopo da Pontormo, Diario "fatto nel tempo che dipingeva il coro di San Lorenzo", 1554-1556, Emilio Cecchi (éd.), Florence, F. Le Monnier, 1956 ; Victor I. Stoichita, Pontormo si manierismul, Bucarest, Editura meridiane, 1978.

39. Jean-Claude Lebensztejn, Le Journal de Jacopo da Pontormo, Paris, éditions Aldines, 1992, p. 149-160.

40. Jean-Claude Lebensztejn, " Note de lecture sur Ewa Lajer-Burcharth, Necklines: The Art of Jacques-Louis David after the Terror ", dans Cahiers du Musée national d'art moderne, $\mathrm{n}^{\circ}$ 75, printemps 2001, p. 112-117.

41. Luís Fróis, Traité sur les contradictions de mœurs entre Européens \& Japonais, Xavier de Castro (trad. fra.), Paris, Éditions Chandeigne, 1993 [éd. orig. : Europa Japao: um dialogo civilizacional no seculo XVI: tratado em que se contem muito sucinta e abreviadamente algumas contradiçoes e diferencas de costumes entre a gente de Europa e esta provincia de Japao..., 1585].

42. Relisant ces propos après tant d'années, j'aimerais ajouter ceci : je regrette que parmi les noms évoqués par mon interlocuteur, d'autres ne soient pas apparus, en particulier celui de Jean Louis Schefer, un des grands penseurs de l'image sous toutes ses formes, et pour qui j'ai toujours eu la plus grande admiration. Enfin, j'ai profité de ma mise à la retraite pour apprendre un peu de chinois, afin de tenter une traduction de Lao-tseu ; elle a été publiée en 2009 par le Théâtre typographique. [J.-Cl. L.] 